advantage to have the knife held in the hand, so that it may be readily lifted and the sections quickly brushed with a goat's-hair brush into a basin of water, or, as in the case of the embryo, quickly drawn one by one from the knife laid flat upon a glass slip. Wach section can thus be readily floated from the knife into a pool of water on the glass slip, so that every part of it is kept in situ. This ready handling of the sections in the mass or singly is impossible with the microtomes devised by Williams and Roy. Commonly the sections are brushed from the knife into a large jar of water, into which the gum diffuses from them. At the end of half an hour or so they are collected with a straight needle in small numbers at a time, and transferred to a second jar of water, in which they are left for the same time. They are then transferred to a third jar, and at the end of half an hour they are transferred in the same way to a stoppered bottle containing methylated spirit, in which they are kept till required for staining and mounting.

The indicator (E, Fig. 1), though useful to the beginner in helping him to make sections of uniform thickness, is soon abandoned, as the manipulator soon learns to regulate the extent of the screw's motion by his sensation. The knife suitable for my instrument requires to be specially made. Both instrument and knife are made by Gardner, instrument maker, South Bridge, Edinburgh.

\section{AN EXAMINATION OF THE SUPPOSED ACTION EXERTED BY THE ANTAGONISM BETWEEN ACIDS AND ALKALIES IN MODIFYING THE TONICITY OF THE HEART AND ARTERIES.}

BY SYDNEY RINGER, M.D., F.P.C.P., PROFESSOR OF MEDICINE IN UNTVERSITY COLLEGE, LONDON; AND

DUDLEY BUXTON, M.D., M.R.C.P. ASSISTANT TO THE PROFESSOR OF MEDICINE IN UNIVERSITY COLLEGE, LONDOX.

THene are two forms of muscular contractility which are called into play during the functional activity of the heart and arteries: the one form is quick, of brief duration; while the other is persistent. This latter form is designated the tonicity of the heart muscle and arterial muscles. Although occurring in the same structure, these forms of contractility offer differences alike in nature and function. It is not our purpose at present to attempt to consider in what directions these differences in nature lie, or to attempt to analyse the mechanism of rhythm. In function, while the quick rhythmic contraction subserves the purpose of propelling the blood, the slow persistent contractility determines the actual capacity of the heart and vessels. Both forms of contractility are influenced by central and peripheral conditions. Thus the arteries can be contracted or dilated by central vaso-motor influences. Again, the arteries are subject to local or peripheral influences, which act either through a hypothetical vaso-motor controlling system situated in the vascular walls, or directly, as by the action of the circulating medium upon the muscular walls of the containing vessels.

Dr. Gaskell has shown ${ }^{1}$ that acids and alkalies, when allowed to circulate in contact with the interior of the heart and arteries, exercise powerful influence upon their tonicity. His experiments are briefly as follows. To ascertain the action of dilute alkaline solutions upon the cardiac muscle, the frog's heart was removed from the body and fixed upon a perfusion cannula. To record the beat and obtain circulation through the heart, Roy's tonometer was used. Normal saline-i.e., 75 per cent. of sodium chloridewas sent through the heart, and caustic soda was then added to the circulating fluid until it was of the strength of 1 in 20,000 salt solution. The heart very slowly and very steadily passed into systole - i.e., a condition of marked tonicity. A similar result followed when the same strength of caustic soda was added to artificial blood solution. Dr. Gaskell says:- "It is clear, then, from these two sets of experiments that a dilute alkaline solution acts upon the muscular tissue of the heart in such a way as to bring it from a position of relaxation to one of contraction, and that, according. to the stage in this alteration of the contractile tissue, so will be the nature of the beat produced by the ventricle; the more the muscle is drawn together by the action of the alkali, the longer is the time of full contraction in each beat, meaning by the term full contraction. the complete closure of the ventricular cavity." The next series of experiments, conducted on precisely similar lines, showed that were an acid-lactic acid, 1 in 20,000-used instead of the caustic soda, that the heart rapidly stopped, remaining in diastole. "The effect, in fact, is exactly the reverse to what took place with the alkaline solution." Dr. Gaskell then undertakes to show that the actions upon the heart of acid and alkali solutions are antagonistic. A heart brought to rest in systole by an alkali was completely relaxed by an acid from a condition of full contraction to one of extreme dilatation. The reverse experiment was also performed; a heart in relaxation by the action of the acid passed into extreme contraction when an. alkaline fluid was circulated. Dr. Gaskell shows further "that alkaline and acid solutions act upon the muscles of the smaller arteries in the same way as upon the cardiac muscle." Dr. Gaskell concludes "that the one factor upon which the state of constriction of the muscles both of the heart and of the arteries depends is the extent of the alkalinity of the fluid surrounding them "; and, again, "one can say, then, that the presence of an allkaline fluid must tend to keep the cardiac and arterial muscles in a state of tonicity, and that upon the extent of the alkalinity the amount of tonicity will, at all events, in part depend." And further, Dr. Gaskell seems to lean towards a theory which would explain varying degrees of tonicity by varying degrees of alkalinity, such being produced by an acid generated during muscular contraction, aided, possibly, by an acid liberated by other structures-e.g., grey matter of the central nervous system during their function. The antagonism exercised upon the heart muscle is certainly proved, but how far the ingenious theory which hangs upon it can be accepted must at present remain unsettled.

We propose to show that substances other than acids may take on a rôle similar to the one performed by acids. 'Thus the salts of potassium can, as we have experimentally proved, act quite similarly to acids, in so far as antagonising the effects of sodium alkaline salts is concermed. 'I'hus, wie found, that when a circulatory finid, composed of simple saline with the addition of canstic soda solution of the strength of 1 in 20,000, was allowed to flow through the heart arranged in the oil chamber of a Roy's tonometer, the heart passed into a condition of persistent systole. If, now, a physiological dose of potassium cllloride-i.e., 1 in 10,000 was added, although the tonicity (spasm) of the heart was lessened, the heart was not enabled to pass into a state of complete dilatation that is, the tonicity persisted to a certain extent. Our next experiments were conducted with the bicarbonate of sodium, this salt being used in place of caustic soda, for the following cogent reasons. Sodtium hydrate, as such, does not exist in the serum or corpuscles of the blood, while bicarbonate of sodium is present in considerable quantity. ${ }^{2}$ Caustic soda, then, possesses properties which, as we shall show in the sequel, are due to attributes other than its alkalinity. A normally beating heart being taken, and the saline solution being circulated through it as before, a quantity of bicarbonate of sorlium was added, equal in strength to 1 in 5000. The effect of this dose was soon apparent. The beat, which had under the influence of the simple saline solution sunk almost to nil, recommenced, and the heart rupirlly passed into a state of persistent systole, induced partly by the" increased tonicity and partly by the fusion of the beats. In subsequent experiments even larger rloses of the soclium bicarbonate were employed, and it was found that the condition of tonicity (persistent spasm) was produced so much the more quickly as was the dose increased.

To return. When the heart was finally contracted a high degree of tonicity being fully established, potassium chloride was added in physiological doses $(1 \mathrm{in} 10,000)$. In every case the ventricle underwent complete relaxation - potassinum chloride completely counteracted the effect of the sodum bicarbonate,toniaity of the heart muscle giving place to relaxation, the rhythmic contractions persisting. To test this antagonism 
further, as lare a quantity of solium bicarbonate as 1 in th) was llwal -i.e., a dose very largely above the proportion of sodium hicarbonate existing in the blood when that fluid is physiological. When the effect of the sodium salt was well matred and a pronounced condition of tonicity was astablished, $3 \mathrm{cc}$ of the potassium salt (1 per cents) were adrled to the 200 cc. of the fluid-i. e., about 1 in 6000 , with the result that complete relaxation of the induced tonicity ensued. Tho addition of $1 \mathrm{cc}$. nore of the potassium chloride, making the proportion about 1 in 5000 , produced no further effect. It is needless to enter in detail into the subsequent experiments; they showed conclusively that : (1) the adlition of the potassium salt can prevent the appearance of tonicity even when large doses of sodium bicarbonate are present; loses far larger than exist in the blood physiologically or could be administered therapeutically; and $(2)$ the tonicity once induced by sodium bicarbonate can be completely overcome, relaxation replacing it when potassium chloride is added in physiological doses.

It would, then, appear from our researches that even if the alkalinity be a factor in promoting cardiac and vascular tonicity, and we shall return to the consideration of that question immediately, it cannot, however be asserted that the presence of an acid or acid tendency is the physiological antagonist in the body whereby cardiac tonicity is lessened or annulled. All that can be asserted, it appears to us, is that the hypothetic production of an acid condition, or rather of a lessened alkaline condition of the blood, may be instrumental in lessening cardiac tonicity.

We have in the experiments narrated above shown that potassium salts can actually prevent the occurrence of cardiac tonicity, even when such a large quantity of alkaline sodium bicarbonate is present as is competent, when unopposed, to induce a condition of extreme cardiac tonicity; and we are warranted by the fact that such potassium salts are present in large quantity in the blood and parenchymatous tissues, in saying that the bicarbonate of sodium cannot be accredited with the power of inducing constriction of the heart and vessels. But in order that we may deal with the problem involved in the action and antagonism between acids and alkalies, it is essential that we should place before us the phyGiological conditions which are present in the blood, and, as far as may be, reproduce them in our experiment. And we must make due allowance in deductions drawn from our results for departures from the physiological standard. It becomes, then, at once evident that the sodium bicarbonate which is found in the plasma could not induce tonicity either in the heart or vessels, its tendency in that direction being completely held in check by the chloride of potassium which, upon Lehmann's, Schmidt's, or Serlini's analyses, ${ }^{3}$ is present in far larger quantities than those employed by us to counteract the sodium bicarbonate. From the above facts and considerations we conclude that variations in the alkalinity of the blood must exercise very little, if any, influence apon the tonicity of the bloodvessels and heart. But with the theory that arterial and cardiac tonus depend upon the alkalinity of the circulating medium is coupled one which would explain rliythm as resulting from an alternating degree of more or less alkalinity. It would then be admitted that our experiments completely subvert such a theory, rendering it wholly untenable.

Further investigations, indeed, have tended to make us believe that the "alkalinity" as such plays a far less important part in the induction of tonicity of the heart and vessels than has been heretofore supposed. Another possible explanation offers itself-riz., that the action of the alkaline bicarbonate of sodium upon the muscle causing the condition called tonicity is at bottom a sodium effect. On this supposition we should expect to find that as the sodium became more or less chemically saturated, so would its effects become less or more marked. And such seems to be the case, for we may, without insisting further upon this point-one really of no important bearing upon the main facts of our present investigation,-instance the behaviour of the hydrate of sodium with the bicarbonate of the same metal.

We further undertook experiments to clear up the question whether the alkalinity was the true factor at work, and we found it was not so. When the heart was supplied with normal saline, massive doses of bicarbonate of sodium were added until the circulating fluid was rendered strongly alkaline, as judged by the bluing of red litmus paper.

${ }^{3}$ Quoted by Gamgee : Physiological Chemistry, p. 69.
Tonicity or persistent spasm was develoued, but when chloride of potassium was adcled the lueart relaxed. An extremely dilute solution of caustic soda ( 1 in 40,000) was then taken. Its reaction to recl litmus paper revealed that it possessed but feeble alkalinity; nevertheless it provoked the condition of persistent spasm or tonicity, and the heart thus treated did not become so completely relaxed, even when large doses of chloride of potassium were added. $\Lambda \mathrm{s}$, however, the blood possesses in its sodium phosphate a possible source of alkalinity other than the sodium bicarbonate, we instituted experiments with that salt. The solution which we employed was strongly alkaline. In physiological quantities we found the phosphate of sodium exercised no influence over the heart muscle, nor were marked results obtained with solutions wlich gave a strongly alkaline reaction. When, however, the sodium phosphate was pushed so that as huge a dose as 1 in 50 of artificial blood solution was reached some fusion with possibly increased tonicity of the heart were apparent. It is highly significant, however, to note that even in this extreme case, the addition of more potassium chloride removed the tendency alike to fusion of beat and increased tonicity of the heart. When the sodium phosphate was used with simple saline solution, we found that for a time it increased the vigour of the beat but produced little increase in cardiac tonicity, and such as appeared was promptly suppressed when potassium chloride in physiological doses was added. These experiments add further evidence that solutions, although strongly alkaline, do not necessarily increase cardiac tonicity. They show also that both the salts of the blood which are usually stated to give to that fluid its alkaline reaction, are completely overridden by potassium chloride, in so far at least as they are operative in inducing an increment in cardiac tonicity.

In experiments the results of which appear in the Transactions of the Royal Medico-Chirurgical Society, vol. Ixvii., p. 78, it was shown that even highly alkaline solutions of the sodium bicarbonate do not as such determine constriction-that is, tonicity of the bloodvessels. Thus, as for the heart so for the bloodvessels, we find there are bodies which promote tonus, and that although some alkaline salts are included among them, yet there are others which oppose tonicity.

To sum up, then, we find from our experiments that the tonicity of the heart and vessels produced by alkaline solutions of sodium salts, such as are present in normal blood, will not only not appear in the presence of an acid, but will not be induced in the presence of potassium chloride; thence it is inconceivable how, in the teeth of the fact that large quantities of this salt-potassium chloride-exist in the blood, the tonicity of the heart and arteries can be due to varying degrees of alkalinity. And we further find the alkalinity of the solution does not determine its power of producing tonicity in the muscles of the heart and smaller vessels, and so we suggest that this result may accrue from the degree of chemical saturation of the sodium present in the sodium salt.

\section{TWO CASES OF GASTROTOMY FOR EXTRA- UTERINE GESTATION.}

BY JAMES BRAITHWATTE, M.D. LOND.,

LECTURER OY DISEASES OF WOMEN AND CHILDREN AT THE LEEDS SCHOOL OF MEDICINE, AND SURGEON TO THE WOMEN AND CRILDREN'S HOSPITAY.

CASE 1.-Mrs. A-_, when three months advanced in her third pregnancy, was seized with severe pelvic pain, so severe that it was neeessary to administer morphia hypodermically. The whole of the remainder of her pregnancy was passed in misery from pain and sickness, with obstinate constipation. Towards the end of the pregnancy her medical attendant, Dr. Green of Leeds, convinced himself that it was a case of abdominal extra-uterine gestation. He could distinctly make out the position of the child through the abdominal walls. I saw the patient with Dr. Green a fortnight after the occurrence of a sanguineous vaginal discharge with some pain, which indicated that the uterus had thrown off the deciduous membrane. I need not say that when this occurs the death of the child may be considered certain. I found the patient in a verv low con- 